\title{
EXTENSIONS OF ISOMETRICALLY INVARIANT MEASURES ON EUCLIDEAN SPACES
}

\author{
PIOTR ZAKRZEWSKI
}

(Communicated by R. Daniel Mauldin)

\begin{abstract}
We consider countably additive, nonnegative, extended real-valued measures that vanish on singletons.

Given a group $G$ of isometries of $\mathbf{R}^{n}$ and a $G$-invariant $\sigma$-finite measure on $\mathbf{R}^{n}$ we study the problem of determining whether it has a proper $G$-invariant extension. We prove that it does, provided that the set of all points with uncountable $G$-orbits is not of measure zero. We also characterize those groups $G$ for which every $G$-invariant $\sigma$-finite measure on $\mathbf{R}^{n}$ has a proper $G$-invariant extension.
\end{abstract}

\section{INTRODUCTION}

In 1935 Szpilrajn [9] proved that the Lebesgue measure on $\mathbf{R}^{n}$ has a proper isometrically invariant extension. In the same paper he quoted Sierpiński who asked if there exists such a maximal extension.

There were many partial solutions to this problem (see [1-3] for the historical details). The complete solution was finally obtained by Ciesielski and Pelc [3], who proved that if $G$ is any group of isometries of $\mathbf{R}^{n}$ containing all translations, then every $G$-invariant $\sigma$-finite measure on $\mathbf{R}^{n}$ has a proper $G$ invariant extension.

In the same paper Ciesielski and Pelc stated the problem of characterizing those groups $G$ of isometries of $\mathbf{R}^{n}$ for which the conclusion of their result remains true.

A few partial solutions to this problem have been obtained. Using ideas of Hulanicki [5], Ciesielski and Pelc [3] proved that if the cardinality of the continuum is small in the sense that there does not exist a $\sigma$-finite measure defined on the power set of $\mathbf{R}$, then no restrictions on $G$ are needed. Next, Ciesielski [1] showed that, in general, it is enough to require that $G$ is transitive, i.e. $\mathbf{R}^{n}$ consists of a single $G$-orbit. This result was proved earlier by Harazišvili under the additional assumption of the Continuum Hypothesis.

Received by the editors December 1, 1988 and, in revised form, November 2, 1989.

1980 Mathematics Subject Classification (1985 Revision). Primary 28C10; Secondary 03 E05.

Key words and phrases. Invariant $\sigma$-finite measure, isometries of $\mathbf{R}^{n}$, real-valued measurable cardinal. 
In the above quoted paper Ciesielski studied the more general question: has a given $G$-invariant $\sigma$-finite measure $m$ on $\mathbf{R}^{n}, G$ being a group of algebraic transformations of $\mathbf{R}^{n}$ (see [1] for the definition), a proper $G$-invariant extension? He proved that it is so, provided that $G$ contains an uncountable subset $H$ with the property that every two different elements of $H$ coincide exactly on a set of measure zero.

Ciesielski asked whether this conclusion remains true unless $m$ is concentrated on a $G$-invariant set $Z \subseteq \mathbf{R}^{n}$ such that the group $\{g \mid Z: g \in G\}$ is countable (see [1, Problem 2]).

In this paper we give an affirmative answer to Ciesielski's question for the case of groups of isometries (Theorem 2.2). We prove that the relevant $G$-invariant set consists exactly of the points with countable $G$-orbits.

As a corollary we obtain a solution to Ciesielski-Pelc's problem (Theorem 2.3). We prove that if the cardinality of the continuum is not small, then the family of all groups $G$ of isometries of $\mathbf{R}^{n}$ with the property that every $G$ invariant $\sigma$-finite measure on $\mathbf{R}^{n}$ has a proper $G$-invariant extension consists exactly of those $G$ 's for which there exists at most one one-element $G$-orbit and all the other $G$-orbits are uncountable.

\section{TERMINOLOGY AND PRELIMINARIES}

Our set-theoretic notation and terminology is standard. Ordinals are identified with sets of their predecessors and cardinals with initial ordinals. In particular $\omega$ denotes the set of natural numbers and the first infinite cardinal. If $A$ is a set, then $P(A)$ denotes the power set of $A$ and $|A|$, the cardinality of $A$. If $f: X \rightarrow Y$ is a function and $A \subset X$, then $f[A]$ denotes the image of $A$ and $f \uparrow A$ the restriction of $f$ to $A$. Symmetric difference of sets $A$ and $B$ is denoted by $A \triangle B$.

By a measure on a set $X$ we mean a countably additive, nonzero function defined on a $\sigma$-algebra of subsets of $X$, assuming values in $[0,+\infty]$ and vanishing on all singletons.

A measure is universal on $X$ if it is defined on $P(X)$. We say that $X$ is large if it carries a universal measure of total measure one; otherwise, $X$ is small. It is well known that $X$ is large if and only if the cardinality of $X$ is at least real-valued measurable (see e.g. [6, p. 300]).

A measure on $X$ is $\sigma$-finite, if $X$ is a countable union of sets of finite measure.

If $m$ is a measure, then $m_{*}$ and $m^{*}$ denote the inner and the outer measure, respectively.

Let $G$ be a group of bijections of a set $X$.

We define:

$G x=\{g(x): g \in G\}$-the $G$-orbit of a point $x \in X$;

$G A=\bigcup_{x \in A} G x$ for $A \subset X$.

We say that $A$ is $G$-invariant if $G A=A$. 
A countable $G$-configuration of a set $N \subset X$ is any set of the form

$$
\bigcup_{n \in \omega} g_{n}[N]
$$

where $\left\{g_{n}: n \in \omega\right\}$ is a countable set of elements of $G$.

A measure $m$ defined on a $\sigma$-algebra $\mathfrak{M}$ of subsets of $X$ is $G$-invariant iff $g[A] \in \mathfrak{M}$ and $m(g[A])=m(A)$ for any $g \in G$ and $A \in \mathfrak{M}$. Notice that the measure completion of a $G$-invariant measure is $G$-invariant. Since we are interested in the existence of extensions of invariant measures, we shall assume, without loss of generality, that all the invariant measures under consideration are complete.

In what follows we shall use some auxiliary results. The first one is the key lemma of Ciesielski [1], formulated here for the case of isometries.

1.1. Lemma. Let $G$ be a group of isometries of $\mathbf{R}^{n}$ containing an uncountable subset $H$. There exists a countable family $\left\{N_{k}: k \in \omega\right\}$ of subsets of $\mathbf{R}^{n}$ satisfying the following conditions:

(i) $\mathbf{R}^{n}=\bigcup_{k \in \omega} N_{k}$;

(ii) for each $k \in \omega$ and every countable $G$-configuration $K$ of $N_{k}$ there exist distinct functions $h_{1}, h_{2} \in H$ such that

$$
h_{1}^{-1}[K] \cap h_{2}^{-1}[K] \subset\left\{x \in \mathbf{R}^{n}: h_{1}(x)=h_{2}(x)\right\} .
$$

For the rest of this section let $G$ be a group of bijections of a set $X$ and let $m$ be a $G$-invariant, $\sigma$-finite measure on $X$.

The methods of extending invariant measures used in the paper are based on the following result of Szpilrajn [9].

1.2. Proposition. If there exists a nonmeasurable subset $N$ of $X$ such that every countable G-configuration of $N$ has inner measure zero, then $m$ has a proper G-invariant extension.

In the proofs we use two easy consequences of the above proposition (cf. Pelc [8]).

1.3. Lemma. If there exists a countable family $\left\{N_{k}: k \in \omega\right\}$ of subsets of $X$ such that

(i) $\mathbf{R}^{n}=\bigcup_{k \in \omega} N_{k}$ and

(ii) for each $k \in \omega$ every countable G-configuration of $N_{k}$ has inner measure zero,

then $m$ has a proper $G$-invariant extension.

1.4. Lemma. If there exists a $G$-invariant non-measurable set $Z \subset X$, then $m$ has a proper G-invariant extension.

The final auxiliary Lemma is essentially due to Harazišvili (see [4]). 
1.5. Lemma. Suppose that $N \subset X$ and for every countable $G$-configuration $K$ of $N$ there exists a countable family $\left\{f_{n}: n \in \omega\right\}$ of elements of $G$ such that $m\left(\bigcap_{n \in \omega} f_{n}[K]\right)=0$. Then every countable G-configuration of $N$ has inner measure zero.

Proof. Suppose otherwise and let $K^{\prime}$ be a countable $G$-configuration of $N$ such that $m_{*}\left(K^{\prime}\right)>0$. Fix a measurable subset $A \subset K^{\prime}$ of positive measure. It follows easily from $\sigma$-finiteness of $m$ that there are countably many functions $\left\{g_{n}: n \in \omega\right\}$ from $G$ such that

$$
A \subset \bigcup_{n \in \omega} g_{n}[A] \text { and } m\left(g\left[\bigcup_{n \in \omega} g_{n}[A]\right] \triangle \bigcup_{n \in \omega} g_{n}[A]\right)=0
$$

for every $g \in G$. Set $K=\bigcup_{n \in \omega} g_{n}\left[K^{\prime}\right] ; K$ is a countable $G$-configuration of $N$ such that for any countable family $\left\{f_{n}: n \in \omega\right\} \subset G$,

$$
m\left(A \backslash \bigcap_{n \in \omega} f_{n}[K]\right)=0 \text {, so } m_{*}\left(\bigcap_{n \in \omega} f_{n}[K]\right)>0 .
$$

This is the desired contradiction.

\section{RESUlts}

Given a group $G$ of isometries of $\mathbf{R}^{n}$, let $L_{G}$ denote the set of all points of $\mathbf{R}^{n}$ with countable $G$-orbits, i.e.:

$$
L_{G}=\left\{x \in \mathbf{R}^{n}:|G x| \leq \omega\right\} .
$$

The basic properties of the set $L_{G}$ are summarized in the following.

\subsection{Lemma.}

(i) $G L_{G}=L_{G}$.

(ii) $\left|\left\{g \mid L_{G}: g \in G\right\}\right| \leq \omega$.

(iii) $L_{G}$ is an affine subspace of $\mathbf{R}^{n}$.

Proof. Part (i) is obvious. To prove the remaining parts let $L$ be the affine subspace of $\mathbf{R}^{n}$ spanned by $L_{G}$. Fix a finite subset $B$ of $L_{G}$ spanning $L$. Notice that the restriction of each $g \in G$ to $L$ is determined by its values on $B$, so the function $g|L \mapsto g| B$ is $1-1$. But, since for each $b \in B,|G b| \leq \omega$ and $B$ is finite, there are only countably many functions $f: B \rightarrow \bigcup_{b \in B} G b$. In particular, $|\{g \mid B: g \in G\}| \leq \omega$, which implies that $|\{g \mid L: g \in G\}| \leq \omega$ proving part (ii). It follows that $|G x| \leq \omega$ for each $x \in L$, which gives $L=L_{G}$ completing the proof of the lemma.

Now we can formulate our main result, which solves Ciesielski's problem. Roughly speaking, a measure extends unless it is concentrated on $L_{G}$. More precisely, the following holds. 
2.2. Theorem. Suppose that $G$ is a group of isometries of $\mathbf{R}^{n}$. Let $m: \mathfrak{M} \rightarrow$ $[0,+\infty]$ be a G-invariant $\sigma$-finite measure on $\mathbf{R}^{n}$. If $m^{*}\left(\mathbf{R}^{n} \backslash L_{G}\right)>0$, then $m$ has a proper $G$-invariant extension.

Proof. Since $L_{G}$ is $G$-invariant, we are done by Lemma 1.4 unless $L_{G} \in \mathfrak{M}$. If this case applies, then $m\left(\mathbf{R}^{n} \backslash L_{G}\right)>0$ and $m_{1}$, defined on $\mathfrak{M}$ by $m_{1}(A)=$ $m\left(A \backslash L_{G}\right)$, is a $G$-invariant $\sigma$-finite measure on $\mathbf{R}^{n}$. To complete the proof it is enough to find a proper $G$-invariant extension of $m_{1}$. Instead, we assume without loss of generality that $m\left(L_{G}\right)=0$.

Let $p$ be the smallest dimension of an affine subspace of positive outer measure. Fix such a subspace $L_{0}$.

Obviously $\left|\left\{g \mid L_{0}: g \in G\right\}\right|>\omega$.

Now consider two cases.

Case 1. $\left|\left\{g\left[L_{0}\right]: g \in G\right\}\right|>\omega$. Then $L_{0}$ satisfies the hypothesis of Lemma 1.5. To see this, let $K=\bigcup_{n \in \omega} g_{n}\left[L_{0}\right]$ be an arbitrary countable $G$-configuration of $L_{0}$ and set $L_{n}=g_{n}\left[L_{0}\right]$ for $n \in \omega ; g_{0}=\mathrm{id}_{\mathbf{R}^{n}}$. For every $n \in \omega$ take $f_{n} \in G$ such that $f_{n}^{-1}\left[L_{n}\right] \neq L_{k}$ for all $k \in \omega$. We have

$$
K \cap \bigcap_{k \in \omega} f_{k}[K] \subset \bigcup_{n, k \in \omega}\left(L_{n} \cap f_{n}\left[L_{k}\right]\right) .
$$

But since $L_{n} \cap f_{n}\left[L_{k}\right] \neq L_{n}$, the set $L_{n} \cap f_{n}\left[L_{k}\right]$ is either empty or is an affine subspace of dimension less than $p$. Accordingly, $m\left(K \cap \bigcap_{k \in \omega} f_{k}[K]\right)=0$.

By the conclusion of Lemma 1.5, every countable $G$-configuration of $L_{0}$ has inner measure zero. At the same time $m^{*}\left(L_{0}\right)>0$, so $L_{0} \notin \mathfrak{M}$ and we are done by Proposition 1.2.

Case 2. $\left|\left\{g\left[L_{0}\right]: g \in G\right\}\right| \leq \omega$. Set $Y=\bigcup_{g \in G} g\left[L_{0}\right]$. If $Y \notin \mathfrak{M}$, then use Lemma 1.4 to extend $m$. Otherwise $m(Y)>0$ and we can assume, without loss of generality, that $m\left(\mathbf{R}^{n} \backslash Y\right)=0$.

Since $\left|\left\{g \mid L_{0}: g \in G\right\}\right|>\omega$, there is an uncountable subset $H$ of $G$ such that $h_{1}\left|L_{0} \neq h_{2}\right| L_{0}$ for any distinct $h_{1}, h_{2} \in H$. Apply Lemma 1.1 to $G$ and $H$ to obtain $\left\{N_{k}: k \in \omega\right\}$. To complete the proof, it is enough to show that the family $\left\{N_{k}: k \in \omega\right\}$ satisfies the hypotheses of Lemma 1.3.

So take an arbitrary $k \in \omega$ and let $K=\bigcup_{n \in \omega} g_{n}\left[N_{k}\right]$ be any countable $G$ configuration of $N_{k}$. By the properties of $\left\{N_{k}: k \in \omega\right\}$ (see Lemma 1.1), there are $h_{1}, h_{2} \in H, h_{1} \neq h_{2}$, such that

$$
h_{1}^{-1}[K] \cap h_{2}^{-1}[K] \cap L_{0} \subset\left\{x \in L_{0}: h_{1}(x)=h_{2}(x)\right\} .
$$

But $h_{1}\left|L_{0} \neq h_{2}\right| L_{0}$, so the set $\left\{x \in L_{0}: h_{1}(x)=h_{2}(x)\right\}$ is either empty or is an affine subspace of dimension less than $p$. Accordingly,

$$
m\left(h_{1}^{-1}[K] \cap h_{2}^{-1}[K] \cap L_{0}\right)=0 .
$$

Let $\left\langle L_{n}: n \in \lambda\right\rangle, \lambda \leq \omega$, be a $1-1$ enumeration of $\left\{g\left[L_{0}\right]: g \in G\right\}$. For each $n \in \lambda$ let $L_{n}=f_{n}\left[L_{0}\right]$; it follows that

$$
m\left(\left(f_{n} \circ h_{1}^{-1}\right)[K] \cap\left(f_{n} \circ h_{2}^{-1}\right)[K] \cap L_{n}\right)=0 .
$$


Consequently,

$$
\begin{aligned}
m^{*}\left(\bigcap_{\substack{k \in \lambda \\
i \in\{1,2\}}}\left(f_{k} \circ h_{i}^{-1}\right)[K]\right) & =m^{*}\left(\bigcap_{\substack{k \in \lambda \\
i \in\{1,2\}}}\left(f_{k} \circ h_{i}^{-1}\right)[K] \cap \bigcup_{n \in \lambda} L_{n}\right) \\
& \leq \sum_{n \in \lambda} m^{*}\left(\bigcap_{\substack{k \in \lambda \\
i \in\{1,2\}}}\left(f_{k} \circ h_{i}^{-1}\right)[K] \cap L_{n}\right) \\
& \leq \sum_{n \in \lambda} m^{*}\left(\left(f_{n} \circ h_{1}^{-1}\right)[K] \cap\left(f_{n} \circ h_{2}^{-1}\right)[K] \cap L_{n}\right)=0
\end{aligned}
$$

and we are done.

As a corollary we obtain a solution to Ciesielski-Pelc's problem.

2.3. Theorem. For a group $G$ of isometries of $\mathbf{R}^{n}$ the following are equivalent:

(i) Every G-invariant $\sigma$-finite measure on $\mathbf{R}^{n}$ has a proper $G$-invariant extension.

(ii) $L_{G}$ is a small set.

(iii) Either $2^{\omega}$ is small or $L_{G}$ is empty or it is a singleton.

Proof. (i) $\rightarrow$ (ii). If $L_{G}$ was large, then there would exist a universal $G$ invariant $\sigma$-finite measure on $\mathbf{R}^{n}$, contradicting (i) (cf. [10]).

(ii) $\rightarrow$ (iii). This is obvious.

$\left(\right.$ iii) $\rightarrow(i)$. If $2^{\omega}$ is small, then use an argument of Ciesielski and Pelc [3]. Otherwise, use Theorem 2.2.

2.4. Remark. The results of this paper essentially reduce the problem of finding necessary and sufficient conditions for extendability of a given $G$-invariant $\sigma$-finite measure on $\mathbf{R}^{n}$ to the case in which $G$ is countable. This case is fully examined in the forthcoming paper of Adam Krawczyk and the author [7].

2.5. Remark. Ciesielski's problem was originally formulated in the more general setting of $G$ being a group of algebraic transformations of $\mathbf{R}^{n}$ (see [1]). We do not know if the answer remains the same in this general case.

\section{ACKNOWLEDGEMENT}

The author wishes to thank Krzysztof Ciesielski for helpful remarks concerning the first version of this paper.

\section{REFERENCES}

1. K. Ciesielski, Algebraically invariant extensions of $\sigma$-finite measures on Euclidean spaces, Trans. Amer. Math. Soc. (to appear).

2. __ How good is Lebesgue measure?, Math. Intelligencer 11 (1989), 54-58. 
3. K. Ciesielski and A. Pelc, Extensions of invariant measures on Euclidean spaces, Fund. Math. 125 (1985), 1-10.

4. A. B. Harazišvili, Groups of transformations and absolutely negligible sets, Bull. Acad. Georgian SSR, 115 (1984). (Russian)

5. A. Hulanicki, Invariant extensions of the Lebesgue measure, Fund. Math. 51 (1962), 111115.

6. T. Jech, Set theory, Academic Press, New York, 1978.

7. A. Krawczyk and P. Zakrzewski, Extensions of measures invariant under countable groups of transformations, Trans. Amer. Math. Soc. (to appear).

8. A. Pelc, Invariant measures and ideals on discrete groups, Dissertationes Math. CCLV (1986), 1-51.

9. E. Szpilrajn, Sur l'extension de la mesure Lebesguienne, Fund. Math. 25 (1935), 551-558.

10. P. Zakrzewski, The existence of universal invariant measures on large sets, Fund. Math. 133 (to appear).

Institute of Mathematics, University of Warsaw, PKiN 9P., 00-901 Warsaw, Poland 